\title{
Determination rate of the Staphylococcus spp. and Candida spp. on skin of children living with HIV infection
}

\author{
Marina Ufimtseva ${ }^{1}$, Kristina Nikolaeva ${ }^{1}$, Alebai Sabitov², Ekaterina Voroshilina ${ }^{3}$, and \\ Natalia Savchenko ${ }^{1}$ \\ ${ }^{1}$ Ural state medical university, Department of Dermatovenereology and Life Safety, 620028, Repin \\ Str., 3, Yekaterinburg, Russian Federation \\ ${ }^{2}$ Ural state medical university, Department of Infectious Diseases and Clinical Immunology of Ural \\ State Medical University, 620028, Repin Str., 3, Yekaterinburg, Russian Federation \\ ${ }^{3}$ Ural state medical university, Department of Microbiology, Virology and Immunology of Ural State \\ Medical University, 620028, Repin Str., 3, Yekaterinburg, Russian Federation
}

\begin{abstract}
On 01.07.2019, more than 1,000 children were diagnosed with HIV infection in the Sverdlovsk region. Studies on the prevalence of dermatoses in HIV-positive children indicate a high incidence of viral, fungal and bacterial infections of the skin, compared with HIV-negative children. The skin is the environment in which a diverse community of microorganisms (microbiota) lives. The Global Human Microbiome Project proved the uniqueness of the human microbiome, and suggests to study the effect of human immunity on the formation of microbiome diversity models. The purpose of this study was to assess the incidence of S. aureus, S. spp., C. albicans, C. glabrata, C. krusei, C. tropicalis / C. parapsilosis as a part of skin microbiota in HIV-positive children by polymerase chain reaction.
\end{abstract}

\section{Introduction}

By the beginning of 2018, more than 10 thousand HIV-positive children were registered in the Russian Federation. By the end of December 2018, 191,882 children from HIV-positive mothers were born in the Russian Federation, 10,822 of them had HIV infection. By July 1, 2019, 1,000 children were diagnosed with HIV infection in the Sverdlovsk Region.

Studies of the prevalence of dermatoses in HIV-infected children indicate a high incidence of viral, fungal and bacterial infections of the skin, compared with HIV-negative children [1]. Dias et al. (2012) determined the profile of dermatoses in children living with HIV at the Fundação de Medicina Tropical do Amazonas and concluded that children with HIV / AIDS have more non-infectious dermatoses than healthy children. According to the authors, atopic dermatitis was most often recorded (22.9\%) [2].

A wide range of hormones, neurotransmitters and cytokines are produced in the skin, which can potentially affect the state of the whole organism. Microbiota of the skin plays an integral role in the differentiation of keratinocytes and in the system of local immunity 
of human skin. For example, a change in the state of microbiota in patients with diabetes, or in patients receiving radiation, systemic glucocorticosteroid, immunosuppressive and cytostatic therapy [3]. Studies have also noted the effect of dysbiosis of the skin microbiome observed in children with primary immunodeficiency on the increase in the number of relapses of skin diseases [4]. The Global Human Microbiome Project explains the uniqueness of the human microbiome and emphasizes the need to study its structure and contribution to the development of human diseases, as well as the effect of immunity on the composition of the microbiome [5].

According to a review by C Navarrete-Dechent et al (2015), the main group of bacteria that cause skin infections are gram-positive species, especially Staphylococcus. The incidence of skin infections caused by Staphylococcus among HIV-positive patients is $8.2 \%$ compared with $3 \%$ in the HIV-negative population [6]. Since the onset of the HIV epidemic, Candida has been identified as a marker of immune deficiency [7]. It is also the most common opportunistic infection. Usually Candida albicans (C. albicans) is detected, but Candida glabrata (C. glabrata), Candida tropicalis (C. tropicalis), Candida krusei (C. krusei) and Candida parapsilosis (C. parapsilosis) are also found [8].

The purpose of the study was to assess the incidence of Staphylococcus aureus, Staphylococcus Spp., Candida albicans, Candida glabrata, Candida krusei, Candida tropicalis / Candida parapsilosis in the skin microbiota in HIV-positive children.

\section{Materials and Methods}

Clinical and laboratory examination of 49 HIV-positive children (18 boys and 31 girls; age range 3 months to 17 years, mean age was $7 \pm 5.1$ ) was carried out. All children were under follow-up care in the State budgetary institution of health care of the Sverdlovsk region "Sverdlovsk Regional Center for the Prevention and Control of AIDS (Table 1).

Table 1. Age and sex characteristics of participants.

\begin{tabular}{|c|c|c|c|c|c|}
\hline Sex & $\begin{array}{c}\text { Breastfeeding } \\
\text { phase } \\
(0-1 \text { y.o. })\end{array}$ & $\begin{array}{c}\text { First } \\
\text { infancy (1- } \\
3 \text { y.o. })\end{array}$ & $\begin{array}{c}\text { Preschool age } \\
\text { (4-6 y.o. })\end{array}$ & $\begin{array}{c}\text { School age } \\
(7-10 \text { y.o. })\end{array}$ & $\begin{array}{c}\text { Puberty age } \\
\text { (11-18 y.o. })\end{array}$ \\
\hline Female & 1 & 1 & 12 & 7 & 10 \\
\hline Male & 0 & 1 & 4 & 6 & 7 \\
\hline
\end{tabular}

According to immunological examination in the $76 \%$ of the presented cases the number of CD4 lymphocytes corresponded to the age norm; moderate immunodeficiency was observed in $5 \%$ of cases, severe immunodeficiency was found in $18 \%$ of children. Patients constantly receiving antiretroviral therapy represented $89 \%$ of the presented cases. Undetectable viral load at the time of the study was achieved in $91 \%$ of children receiving therapy for 24 weeks or more. The perinatal transmission of HIV-infection was established in all children.

A total of 65 samples of skin swabs were obtained from HIV-positive children. From the intact interscapular region, 49 swabs were obtained. In the case of dermatosis, an additional swab was taken from the skin lesion, thus, 5 children were swabbed from two locations, and one child from three.

Material for research was collected in a micro-centrifuge tube containing physiological salt solution. Storage and transportation of material was carried out in accordance with current regulatory documents [9]. All 65 samples were examined for presence of 
Staphylococcus spp. and Candida spp.. DNA of C. albicans, C. glabrata, C. krusei, C. tropicalis / C. parapsilosis, Staphylococcus spp., S. aureus was detected by real-time PCR (RT-PCR) using reagent kits for scientific use and DTPrime 4M1 real-time PCR instrument according to the manufacturer's instructions (DNA-Technology, Russia).

The obtained data was analysed using Microsoft Excel 2016 (Microsoft, USA) and Fisher's combined probability test. Results were regarded as statistically significant, in case $\mathrm{P}<0.05$.

Patient Informed Consent Form for research, diagnostic and therapeutic measures has been developed (or legally authorized representative in case of a patient who has not reached the age established by part 2 of article 54 of the Federal Law of November 21, 2011 No. 323-FZ). The study was approved by the Ethics Committee of the Ural State Medical University (Protocol No. 10 from 12.20.2019).

\section{Results and Discussion}

Clinical examination revealed skin diseases in 19 (38.8\%) of 49 children, including 8 (44.4\%) of 18 boys and $11(35.5 \%)$ of 31 girls.

In $8(16.3 \%)$ of 49 children, chronic dermatoses were detected, including atopic dermatitis in 3 cases $(6 \%)$, seborrheic dermatitis - 2 (2\%), skin xerosis - $2(2 \%)$, follicular hyperkeratosis - $1(2 \%)$. Infectious skin diseases were reported in $11(20 \%)$ children, including $4(8.1 \%)$ bacterial (crevice impetigo, streptoderma), 4(8.1\%) - fungal (oral candidiasis, scalp microsporia) and $3(6.1 \%)$ - viral (herpes catarrhalis, viral warts). In 5 patients, two or more dermatoses were revealed: combinations of oral candidiasis with herpes catarrhalis, streptoderma, crevice impetigo, and seborrhea of the scalp were found; viral warts were registered in children with xerosis. There was no statistically significant dependence of the severity of the dermatosis on the degree of immunodeficiency and viral load.

Staphylococcus spp. detected in $18(36.7 \%)$ samples, including children without skin pathology in 9 (33.3\%) of 30 cases and children with chronic dermatoses in $9(47.3 \%)$ of 19 cases. S. aureus was identified in 4 samples, three of them were obtained from skin lesions (crevice impetigo, streptococcal impetigo of the nasolabial triangle, oral candidiasis). Candida was found in all samples obtained from affected skin areas. C. non-albicans (C. krusei, C. tropicalis / C. parapsilosis), resistant to standard antimycotic drugs, were detected in $2(29 \%)$ cases.

Table 2. The results of PCR-diagnostics of samples.

\begin{tabular}{|l|c|c|c|c|c|c|}
\hline \multirow{2}{*}{ Group of patients } & \multicolumn{5}{|c|}{ Detectable microorganisms } \\
\cline { 2 - 7 } & S.spp. & S.aureus & C.albicans & C.glabrata & C.krusei $\begin{array}{c}\text { C.tropicalis/ } \\
\text { C.parapsilosis }\end{array}$ \\
\hline $\begin{array}{l}1 \text { HIV+ GROUP } \\
\text { (skin diseases, } \\
19 \text { people) }\end{array}$ & $47,3 \%$ & $5,3 \%$ & $31,5 \%$ & $0 \%$ & $5,3 \%$ & $10,5 \%$ \\
\hline $\begin{array}{l}2 \text { HIV+ GROUP } \\
\text { (intact skin, } \\
30 \text { people) }\end{array}$ & $33,3 \%$ & $10 \%$ & $3,3 \%$ & $0 \%$ & $0 \%$ & $0 \%$ \\
\hline
\end{tabular}


Our results demonstrate increased bacterial and fungal colonization of the skin, as well as a greater variety of Candida non-albicans species in HIV-positive children, when opportunistic microorganisms can cause infectious dermatoses.

Although no analysis of antimycotic drug administration among sample has been performed, the detection of Candida non-albicans may be associated with the use of fluconazole, an antifungal drug with low toxicity, which is widely used in the prevention and treatment of candidiasis in children. According to the literature, fluconazole can lead to the resistance of $\mathrm{C}$. albicans and to the spread of Candida species less susceptible to flucanazole, such as C. glabrata, C. tropicalis, and C. krusei [8].

In addition, co-infection of certain species of C. albicans and S. aureus can lead to infectious synergies with increased toxicity and an enhanced inflammatory response [10].

According to CH Shiboski et al. (2011) infectious dermatoses in HIV-infected children are more severe and have a longer course than in children with intact immunity, however, a detailed analysis of the risk factors for infectious dermatoses in HIV-infected patients was not performed [11]. Moreover, dermatoses in HIV-infected children are resistant to standard methods of therapy, which makes it necessary to study the microbiota of the skin and its interaction with factors of the innate and acquired immune response.

The limitations of this study include the small sample size of patients and the absence of a control group, and therefore it is planned to continue studying the structure of the skin microbiota with expanding the spectrum of the analyzed microorganisms, increasing the group of HIV-positive children and then comparing the results with the control group of HIV-negative children.

\section{Conclusion}

1. We have found that in HIV-positive children C. krusei, C. tropicalis / C. parapsilosis, resistant to standard antimycotic treatment, and Staphylococcus spp. is persistant.

2. The presence of opportunistic staphylococci and yeast-like fungi in the skin microbiota and the greater variety of Candida spp. species observed in children living with HIV infection may be the main cause of frequent relapses of skin diseases, despite an undetectable viral load and the absence of immunodeficiency.

3. Detailed information on the structure of the skin microbiota and its interaction with immunity factors may be useful in creating specialized products for basic skin care for atrisk groups of patients. If the methods of external therapy aimed at eliminating pathogenic agents are supplemented with methods that support the maintenance of microbial homeostasis, the risk of developing skin diseases caused by resistant bacteria can be reduced.

\section{References}

1. B. Duko, E. Geja, M.Zewude, S. Mekonen, BMC Res Notes, 11, 1 (2018)

2. E.D. Dias, G. Cunha Mda, S. Talhari, An Bras Dermatol, 87, 3 (2012)

3. M. Muszer, M. Noszczyńska, K. Kasperkiewicz, M. Skurnik, Arch. Immunol. Ther. Exp. (Warsz), 63, 4, (2015)

4. J. Oh, A.F. Freeman, Genome Res., 23, 12 (2013)

5. L.M. Proctor, H.H. Creasy, J.M. Fettweis, Nature, 569, 641-648 (2019)

6. J.E. Gallant, D. Moore, R.E. Chaisson, Ann. Intern. Med., 122, 9, 730-731 (1995)

7. C. Navarrete-Dechent, R. Ortega, F. Fich, M. Concha, Rev Chilena Infectol, 32, 1 (2015)

8. P.F.Wu, W.L. Liu, M.H. Hsieh, Emerg. Microbes Infect., 6, 10 (2017) 
9. Methodical instructions MU 4.2.2039-05 "Technique for collecting and transporting biomaterials to microbiological laboratories" (approved and put into effect by the Chief State Sanitary Doctor of the Russian Federation on December 23, 2005)

10. B.M. Peters, M.C. Nover, Infect. Immun., 81, 6 (2013)

11. C.H. Shiboski, J.Y. Webster-Cyriaque, M. Ghannoum, J.S. Greenspan, D. Dittmer, Adv. Dent. Res., 23, 1, 28-33 (2011) 